Proyecciones Journal of Mathematics

Vol. 31, No 3, pp. 247-259, September 2012.

Universidad Católica del Norte

Antofagasta - Chile

\title{
On the spectral radius of weighted digraphs
}

\author{
S. BURCU BOZKURT \\ SelÇUk University, Turkey \\ and \\ DURMUS BOZKURT \\ Selçuk University, Turkey \\ Received: March 2012. Accepted : June 2012
}

\begin{abstract}
We consider the weighted digraphs in which the arc weights are positive definite matrices. We obtain some upper bounds for the spectral radius of these digraphs and characterize the digraphs achieving the upper bounds. Some known upper bounds are then special cases of our results.
\end{abstract}

Keywords : Weighted digraph, spectral radius, upper bound.

Subjclass : [2000] 05C50, 05C10. 


\section{Introduction}

We consider digraphs which have no loops or multi-arcs. Let $G=(V, E)$ be a strongly connected digraph on the vertex set $V=\{1,2, \ldots, n\}$. If $(i, j)$ is an $\operatorname{arc}$ of $G$, then $i$ is called the initial vertex and $j$ is called the terminal vertex of this arc. The outdegree $d_{i}^{+}$of a vertex $i$ in the digraph $G$ is defined to be the number of arcs in $G$ with initial vertex $i$. Let $t_{i}^{+}$ be the sum of the outdegrees of all vertices in $N_{i}^{+}=\{j:(i, j) \in E\}$ and call it the 2-outdegree. Moreover, set $m_{i}^{+}:=\frac{t_{i}^{+}}{d_{i}^{+}}$, the average 2-outdegree, $1 \leq i \leq n$.

A weighted digraph is a digraph in which each arc is assigned a weight, similarly to a weighted graph. It is called a network in the context of the graph theory. In the literature, especially in control theory, quantum mechanics etc., the entries of matrices are also matrices have been studied. Considering these, we define the generalized weighted digraph as a digraph in which each arc is assigned a square matrix which is called a weight matrix. Throughout this paper, unless otherwise stated, all weight matrices will be assumed positive definite. It is clear that the weight of the arc can also be positive numbers. Moreover if each arc of weighted digraph bearing weight 1 , then it is simply an (unweighted) digraph.

Now we introduce some notations. Let $G=(V, E)$ be a generalized weighted digraph on the vertex set $V=\{1,2, \ldots, n\}$ and let $\left(W_{i j}\right)$ be the positive definite weight matrix of order $p$ of the $\operatorname{arc}(i, j) \in E$. Let $W_{i}^{+}=$ $\sum_{j \in N_{i}^{+}} W_{i j}$ be the outweight matrix of the vertex $i$ and let $d(i, j)$ be the distance, i.e., length of a shortest directed $i-j$ path [3] from the vertex $i$ to $j$ in $G$.

The adjacency matrix of a weighted digraph $G$ is defined as the block matrix $A(G)=\left(a_{i j}\right)$, where

$$
a_{i j}=\left\{\begin{array}{cc}
W_{i j} & \text { if }(i, j) \in E \\
0 & \text { otherwise }
\end{array}\right.
$$

Here zero denotes the $p \times p$ zero matrix. Thus $A(G)$ is a square matrix of order $n p$. The eigenvalues $\rho_{1}, \rho_{2}, \ldots, \rho_{n p}$ of $G$ are the eigenvalues of its adjacency matrix $A(G)$. In general $A(G)$ is not Hermitian and so its eigenvalues can be complex numbers. We usually arrange them in descending order: $\left|\rho_{1}\right| \geq\left|\rho_{2}\right| \geq \cdots \geq\left|\rho_{n p}\right|$. The spectral radius of $G$ is defined as its largest eigenvalue in modulus. More generally, if $B$ is a square matrix, we use $\rho_{1}(B)$ to denote the spectral radius of the matrix $B$. 
Now we define outweight regular and outweight semi-regular digraphs. For this let $G=(V, E)$. If the vertex set $V$ is partitioned into two nonempty sets $V_{1}$ and $V_{2}$ such that every vertex $i$ in $V_{1}$ has the same $\rho_{1}\left(W_{i}^{+}\right)$and every vertex $j$ in $V_{2}$ has the same $\rho_{1}\left(W_{j}^{+}\right)$, then $G$ is called an outweight semi-regular digraph. If $\rho_{1}\left(W_{i}^{+}\right)=\rho_{1}\left(W_{j}^{+}\right)$in outweight semi-regular digraph, then $G$ is called an outweight regular digraph.

The spectral radius of unweighted digraphs and its bounds have been studied in $[1,2,4,5,11,12]$. In this paper, we study the spectral radius of the generalized weighted digraphs where the arc weights are positive definite matrices. We obtain some upper bounds for the spectral radius of these digraphs and characterize digraphs that achieve the upper bounds. Some known upper bounds are then special cases of our results.

In fact, for undirected weighted graphs the following results have been obtained in [6] and [7].

Theorem 1.1. [6] Let $G=(V, E)$ be a simple connected weighted graph. Then

$$
\left|\rho_{1}\right| \leq \max _{(i, j) \in E}\left\{\sqrt{\sum_{k \in N_{i}} \rho_{1}\left(W_{i k}\right) \sum_{k \in N_{j}} \rho_{1}\left(W_{j k}\right)}\right\}
$$

where $W_{i j}$ is the positive definite weight matrix of order $p$ of the edge $(i, j) \in E$. Moreover equality holds in e1 if and only if

(i) $G$ is a weight regular graph or $G$ is a weight semi-regular bipartite graph;

(ii) $\left\{W_{i j}\right\}_{i, j=1, \ldots, n}$ have a common eigenvector corresponding to the largest eigenvalue $\rho_{1}\left(W_{i j}\right)$ for all $i, j$.

Theorem 1.2. [7] Let $G=(V, E)$ be a simple connected weighted graph. Then

$$
\left|\rho_{1}\right| \leq \max _{i \in V}\left\{\sqrt{\sum_{j \in N_{i}} \rho_{1}\left(W_{i j}\right) \sum_{k \in N_{j}} \rho_{1}\left(W_{j k}\right)}\right\}
$$

where $\left(W_{i j}\right)$ is the positive definite weight matrix of order $p$ of the edge $i j \in E$. Moreover equality holds in e2 if and only if

(i) $G$ is a weight regular graph or $G$ is a weight semi-regular bipartite graph; 
(ii) $\left\{W_{i j}\right\}_{i, j=1, \ldots, n}$ have a common eigenvector corresponding to the largest eigenvalue $\rho_{1}\left(W_{i j}\right)$ for all $i, j$.

We will give generalizations of Theorem 1.2 for the spectral radius of weighted digraphs, by following the idea in [7]. The terminology not defined in here can be found in $[1,2,6,7,8,9]$.

\section{Upper bounds for the spectral radius of weighted digraphs}

We first recall some lemmas.

Lemma 2.1. [10] Let $B$ be an $n \times n$ Hermitian matrix with $\rho_{1}$ as eigenvalue with largest modulus. Then for any $x \in C^{n}(x \neq 0), y \in C^{n}(y \neq 0)$ the spectral radius $\left|\rho_{1}\right|$ satisfies

$$
\left|x^{*} B y\right| \leq\left|\rho_{1}\right| \sqrt{x^{*} x} \sqrt{y^{*} y}
$$

Moreover the equality holds in e3 if and only if $x$ is an eigenvector of $B$ corresponding to $\rho_{1}$ and $y=\alpha x$ for some $\alpha \in R$.

Lemma 2.2. [6] Let $B_{1}, B_{2}, \ldots, B_{k}$ be positive definite matrices of order $n$ and let $B=\sum_{i=1}^{k} B_{i}$. If $x \in C^{n}$ is an eigenvector of each $B_{i}$ corresponding to the largest eigenvalue $\rho_{1}\left(B_{i}\right)$ for all $i$, then $x$ is also an eigenvector of $B$ corresponding to the largest eigenvalue $\rho_{1}(B)$.

From Lemma 2.2, we can give the following lemma for weighted digraphs without proof.

Lemma 2.3. Let $G=(V, E)$ be a weighted digraph and let $\left(W_{i j}\right)$ be the positive definite weight matrix of order $p$ of the arc $(i, j) \in E$ and $W_{i}^{+}=\sum_{j \in N_{i}^{+}} W_{i j}$. Also let $x$ be an eigenvector of $W_{i j}$ corresponding to the largest eigenvalue $\rho_{1}\left(W_{i j}\right)$ for all $i, j$. Then $x$ is also an eigenvector of $W_{i}^{+}$ corresponding to the largest eigenvalue $\rho_{1}\left(W_{i}^{+}\right)$for all $i$, and $\rho_{1}\left(W_{i}^{+}\right)=$ $\sum_{j \in N_{i}^{+}} \rho_{1}\left(W_{i j}\right)$.

For the spectral radius of weighted digraphs, we obtain the following upper bounds. 
Theorem 2.4. Let $G=(V, E)$ be a strongly connected weighted digraph. Then

$$
\left|\rho_{1}\right| \leq \max _{i \in V}\left\{\sqrt{\sum_{j \in N_{i}^{+}} \rho_{1}\left(W_{i j}\right) \sum_{k \in N_{j}^{+}} \rho_{1}\left(W_{j k}\right)}\right\}
$$

where $\left(W_{i j}\right)$ is the positive definite weight matrix of order $p$ of the arc $(i, j) \in E$. Moreover, the equality holds in newbound if and only if

(i) $G$ is an outweight regular digraph or $G$ is an outweight semi-regular digraph;

(ii) $\left\{W_{i j}\right\}_{i, j=1, \ldots, n}$ have a common eigenvector corresponding to the largest eigenvalue $\rho_{1}\left(W_{i j}\right)$ for all $i, j$.

Proof. Let $X$ be an eigenvector corresponding to eigenvalue $\rho_{1}\left(\left|\rho_{1}\right|\right.$ is the spectral radius) of $A(G)$. Now we assume that $x_{i_{1}}$ is the vector component of $X$ such that $x_{i_{1}}^{*} x_{i_{1}}=\max _{k \in V}\left\{x_{k}^{*} x_{k}\right\}$. Since $X$ is nonzero, so is $x_{i_{1}}$. We have

$$
A(G) X=\rho_{1} X .
$$

From the $i_{1}$ th equation of e5, we have

$$
\rho_{1} x_{i_{1}}=\sum_{k \in N_{i_{1}}^{+}} W_{i_{1} k} x_{k}
$$

i.e., $\rho_{1} x_{i_{1}}^{*} x_{i_{1}}=\sum_{k \in N_{i_{1}}^{+}} x_{i_{1}}^{*} W_{i_{1} k} x_{k}$. Taking modulus on both sides of e6 and using Lemma 2.1, we get

$$
\begin{aligned}
\left|\rho_{1}\right| x_{i_{1}}^{*} x_{i_{1}} & =\left|\sum_{k \in N_{i_{1}}^{+}} x_{i_{1}}^{*} W_{i_{1} k} x_{k}\right| \leq \sum_{k \in N_{i_{1}}^{+}}\left|x_{i_{1}}^{*} W_{i_{1} k} x_{k}\right| \\
& \leq \sum_{k \in N_{i_{1}}^{+}} \rho_{1}\left(W_{i_{1} k}\right) \sqrt{x_{i_{1}}^{*} x_{i_{1}}} \sqrt{x_{k}^{*} x_{k}} .
\end{aligned}
$$

Since $x_{i_{1}}^{*} x_{i_{1}} \neq 0$,

$$
\left|\rho_{1}\right| \sqrt{x_{i_{1}}^{*} x_{i_{1}}} \leq \sum_{k \in N_{i_{1}}^{+}} \rho_{1}\left(W_{i_{1} k}\right) \sqrt{x_{k}^{*} x_{k}}
$$


For all $k$ such that $x_{k} \neq 0$ and $k \in N_{i_{1}}^{+}$,

$$
\left|\rho_{1}\right| \sqrt{x_{k}^{*} x_{k}} \leq \sum_{r \in N_{k}^{+}} \rho_{1}\left(W_{k r}\right) \sqrt{x_{r}^{*} x_{r}}
$$

i.e.,

$$
\left|\rho_{1}\right| \sqrt{x_{k}^{*} x_{k}} \leq \sqrt{x_{i_{1}}^{*} x_{i_{1}}} \sum_{r \in N_{k}^{+}} \rho_{1}\left(W_{k r}\right) .
$$

Multiplying both sides of e9 by $\left|\rho_{1}\right|$ and using e11 we obtain

$$
\left|\rho_{1}\right| \leq \sqrt{\sum_{k \in N_{i_{1}}^{+}} \rho_{1}\left(W_{i_{1} k}\right) \sum_{r \in N_{k}^{+}} \rho_{1}\left(W_{k r}\right)} .
$$

Hence the upper bound newbound follows. Now we suppose that the equality holds in newbound. Then all inequalities in the above argument must be equalities. If there exists $k, k \in N_{i_{1}}^{+}$such that $x_{k}=0$, therefore from e9 and e12 we have $\left|\rho_{1}\right|<\sqrt{\sum_{k \in N_{i_{1}}^{+}} \rho_{1}\left(W_{i_{1} k}\right) \sum_{r \in N_{k}^{+}} \rho_{1}\left(W_{k r}\right)}$

which contradicts e12. Thus $x_{k} \neq 0, k \in N_{i_{1}}^{+}$. From e8 and using Lemma 2.1 , we conclude that both $x_{i_{1}}$ and $x_{k}$ are the eigenvectors of $W_{i_{1} k}$ corresponding to the largest eigenvalue $\rho_{1}\left(W_{i_{1} k}\right)$ for all $k, k \in N_{i_{1}}^{+}$. Hence for any $k, k \in N_{i_{1}}^{+}, x_{k}=c_{i_{1}, k} x_{i_{1}}$ for some $c_{i_{1}, k}$. Since $W_{i_{1} k}$ is a positive definite matrix and $x_{i_{1}}$ is an eigenvector of $W_{i_{1} k}$ corresponding to the largest eigenvalue $\rho_{1}\left(W_{i_{1} k}\right)$, we have

$$
x_{i_{1}}^{*} W_{i_{1} k} x_{i_{1}}>0
$$

From (7), as $x_{k}=c_{i_{1}, k} x_{i_{1}}$

$$
\begin{aligned}
\left|\sum_{k \in N_{i_{1}}^{+}} c_{i_{1}, k} x_{i_{1}}^{*} W_{i_{1} k} x_{i_{1}}\right| & =\sum_{k \in N_{i_{1}}^{+}}\left|c_{i_{1}, k}\right|\left|x_{i_{1}}^{*} W_{i_{1} k} x_{i_{1}}\right| \\
& =\sum_{k \in N_{i_{1}}^{+}}\left|c_{i_{1}, k}\right| x_{i_{1}}^{*} W_{i_{1} k} x_{i_{1}}
\end{aligned}
$$

i.e., $c_{i_{1}, k}>0$ for all $k \in N_{i_{1}}^{+}$, and the last equality follows from e13. Similar to above discussions, from equality in e10, we conclude that both $x_{k}$ and $x_{r}$ are the eigenvectors of $W_{k r}$ corresponding to the largest eigenvalue $\rho_{1}\left(W_{k r}\right)$ 
for all $r, r \in N_{k}^{+}, k \in N_{i_{1}}^{+}$. Therefore for any $r, r \in N_{k}^{+}, k \in N_{i_{1}}^{+}, x_{r}=c_{k, r} x_{k}$ for some $c_{k, r}>0$. From e11 we get

$$
x_{r}^{*} x_{r}=x_{i_{1}}^{*} x_{i_{1}} \text { for } r \in N_{k}^{+}, k \in N_{i_{1}}^{+}
$$

i.e., $c_{k, r}^{2} c_{i_{1, k}}^{2}=1$ as $x_{k}=c_{i_{1}, k} x_{i_{1}}$ and $x_{r}=c_{k, r} x_{k}$ and $x_{i_{1}} \neq 0$. In other words $c_{k, r} c_{i_{1}, k}=1$ by $c_{i_{1}, k}>0$ and $c_{k, r}>0$. Thus $x_{r}=c_{k, r} c_{i_{1}, k} x_{i_{1}}=x_{i_{1}}$ for all $r, r \in N_{k}^{+}, k \in N_{i_{1}}^{+}$, i.e., $x_{r}=x_{i_{1}}$, for all $r, r \in N_{N_{i_{1}}^{+}}$where $N_{N_{i_{1}}^{+}}=\left\{r: r \in N_{k}^{+}, k \in N_{i_{1}}^{+}, r \neq i_{1}\right\}$. Similarly, we can show that $x_{s}=x_{i_{1}}$ for all $s, s \in N_{N_{r}^{+}}, r \in N_{N_{i_{1}}^{+}}$. Continuing the procedure, since $G$ is strongly connected, it is easy to see that $x_{i}=x_{i_{1}}$ for $d\left(i_{1}, i\right)$ even. We denote by $V_{1}=\left\{i: x_{i}=x_{i_{1}}\right\}$ and $V_{2}=V \backslash V_{1}$. We first assume that there is an arc $(i, j) \in E\left(V_{1}\right)$, where $E\left(V_{1}\right)$ denotes the set of arcs in $V_{1}$. We have $x_{i}=x_{j}=x_{i_{1}}$. Therefore

$$
x_{r}=x_{i_{1}} \text { for all } r, r \in N_{j}^{+}, j \in N_{i}^{+}
$$

and

$$
x_{r}=x_{i_{1}} \text { for all } r, r \in N_{i}^{+}, i \in N_{j}^{+} .
$$

Using the same technique as above, since $G$ is strongly connected, we conclude that $x_{i}=x_{i_{1}}$ for all $i \in V$. Furthermore, $x_{i_{1}}$ is an eigenvector of $W_{i j}$ corresponding to the largest eigenvalue of $\rho_{1}\left(W_{i j}\right)$ for all $i, j$. From Lemma 2.3, $x_{i_{1}}$ is also an eigenvector of $W_{i}^{+}$corresponding to the largest eigenvalue of $\rho_{1}\left(W_{i}^{+}\right)$for all $i$. For $i \in V$

$$
\rho_{1} x_{i_{1}}=\sum_{k \in N_{i}^{+}} W_{i, k} x_{i_{1}}=W_{i}^{+} x_{i_{1}}=\rho_{1}\left(W_{i}^{+}\right) x_{i_{1}} .
$$

For $i, k \in V$

$$
\rho_{1} x_{i_{1}}=\rho_{1}\left(W_{i}^{+}\right) x_{i_{1}}=\rho_{1}\left(W_{k}^{+}\right) x_{i_{1}}
$$

i.e., $\left(\rho_{1}\left(W_{i}^{+}\right)-\rho_{1}\left(W_{k}^{+}\right)\right) x_{i_{1}}=0$. Since $x_{i_{1}} \neq 0, \rho_{1}\left(W_{i}^{+}\right)$is constant for all $i \in V$. Hence $G$ is an outweight regular digraph. Next we assume that there is no arc in the set $E\left(V_{1}\right)$. So there is an arc from the each vertex in $V_{1}$ to the vertices in the set $V_{2}$. From above for any $i \in V_{1}$

$$
x_{r}=x_{i_{1}} \text { for all } r, r \in N_{j}^{+}, j \in N_{i}^{+} .
$$


Thus we can conclude that there is an arc from the each vertex in $V_{2}$ to the vertices in the set $V_{1}$ since $G$ is strongly connected. Hence $G$ is bipartite. Now we assume that all the eigen-components corresponding to the vertices in $V_{2}$ are not equal. Let $x_{j_{1}}^{*} x_{j_{1}}=\max _{r \in V_{2}}\left\{x_{r}^{*} x_{r}\right\}$. Then there exists a vertex $k \in V_{2}$ such that $x_{k}^{*} x_{k}<x_{j_{1}}^{*} x_{j_{1}}, j_{1}, k \in V_{2} ; k, j_{1} \in N_{i}^{+}$, $i \in V_{1}$.

From e11 and e9 we obtain

$$
\left|\rho_{1}\right| \sqrt{x_{j_{1}}^{*} x_{j_{1}}}=\sqrt{x_{i_{1}}^{*} x_{i_{1}}} \sum_{r \in N_{j_{1}}^{+}} \rho_{1}\left(W_{j_{1} r}\right)
$$

and

$$
\left|\rho_{1}\right| \sqrt{x_{i_{1}}^{*} x_{i_{1}}}=\sum_{s \in N_{r}^{+}} \rho_{1}\left(W_{r s}\right) \sqrt{x_{s}^{*} x_{s}}, r \in N_{j_{1}}^{+} .
$$

Therefore

$$
\left|\rho_{1}\right|^{2} \sqrt{x_{j_{1}}^{*} x_{j_{1}}}=\sum_{r \in N_{j_{1}}^{+}} \rho_{1}\left(W_{j_{1}, r}\right) \sum_{s \in N_{r}^{+}} \rho_{1}\left(W_{r s}\right) \sqrt{x_{s}^{*} x_{s}},
$$

i.e.,

$$
\left|\rho_{1}\right|<\sqrt{\sum_{r \in N_{j_{1}}^{+}} \rho_{1}\left(W_{j_{1}, r}\right) \sum_{s \in N_{r}^{+}} \rho_{1}\left(W_{r s}\right)}
$$

which is a contradiction. So $x_{j}=x_{j_{1}}$ for all $j \in V_{2}$. Hence $V_{1}=\left\{i: x_{i}=x_{i_{1}}\right\}$ and $V_{2}=\left\{i: x_{i}=c x_{i_{1}}, c>0\right\}$. Moreover, $x_{i_{1}}$ is an eigenvector of $W_{i j}$ corresponding to the largest eigenvalue $\rho_{1}\left(W_{i j}\right)$ for all $i, j$. By Lemma 2.3, $x_{i_{1}}$ is also an eigenvector of $W_{i}^{+}$corresponding to the largest eigenvalue $\rho_{1}\left(W_{i}^{+}\right)$for all $i$. For $i \in V_{1}$,

$$
\rho_{1} x_{i_{1}}=\sum_{k \in N_{i}^{+}} W_{i k} x_{k}=c W_{i}^{+} x_{i_{1}}=c \rho_{1}\left(W_{i}^{+}\right) x_{i_{1}} .
$$

For $i, k \in V_{1}$,

$$
\rho_{1} x_{i_{1}}=c \rho_{1}\left(W_{i}^{+}\right) x_{i_{1}}=c \rho_{1}\left(W_{k}^{+}\right) x_{i_{1}},
$$

i.e., $\rho_{1}\left(W_{i}^{+}\right)=\rho_{1}\left(W_{k}^{+}\right)$since $x_{i_{1}}$ is nonzero and $c \neq 0$. Hence $\rho_{1}\left(W_{i}^{+}\right)$ is constant for all $i \in V_{1}$. Similarly we can show that $\rho_{1}\left(W_{j}^{+}\right)$is constant for all $j \in V_{2}$. Hence $G$ is an outweight semi-regular digraph. 
Conversely, we suppose that the conditions (i)-(ii) shown in the second part of the theorem hold for the digraph $G$. Then we must prove that

$$
\left|\rho_{1}\right|=\max _{i \in V} \sqrt{\sum_{j \in N_{i}^{+}} \rho_{1}\left(W_{i j}\right) \sum_{k \in N_{j}^{+}} \rho_{1}\left(W_{j k}\right)} .
$$

Let $x$ be a common eigenvector of $\left(W_{i j}\right)$ corresponding to the largest eigenvalue $\rho_{1}\left(W_{i j}\right)$ for all $i, j$. Using Lemma 2.3 , each $W_{i}^{+}$also has eigenvector $x$ corresponding to the largest eigenvalue of $\rho_{1}\left(W_{i}^{+}\right)$. First we suppose that $G$ is an outweight semi-regular digraph. Let $V_{1}, V_{2}$ be the partite sets of $G$. Also let $\rho_{1}\left(W_{i}^{+}\right)=a$ for $i \in V_{1}$ and $\rho_{1}\left(W_{i}^{+}\right)=b$ for $i \in V_{2}$. Then the following equation can be easily seen:

$\sqrt{a b}\left(\begin{array}{c}x \\ x \\ \vdots \\ x \\ \sqrt{\frac{b}{a}} x \\ \sqrt{\frac{b}{a}} x \\ \vdots \\ \sqrt{\frac{b}{a}} x\end{array}\right)=\left(\begin{array}{ccc|ccc}0 & \ldots & 0 & W_{1, k+1} & \ldots & W_{1, n} \\ 0 & \ldots & 0 & W_{2, k+1} & \ldots & W_{2, n} \\ \vdots & & \vdots & \vdots & & \vdots \\ 0 & \ldots & 0 & W_{k, k+1} & \ldots & W_{k, n} \\ \hline W_{k+1,1} & \ldots & W_{k+1, k} & 0 & \ldots & 0 \\ W_{k+2,1} & \ldots & W_{k+2, k} & 0 & \ldots & 0 \\ \vdots & & \vdots & \vdots & & \vdots \\ W_{n, 1} & \ldots & W_{n, k} & 0 & \ldots & 0\end{array}\right)\left(\begin{array}{c}x \\ x \\ \vdots \\ x \\ \sqrt{\frac{b}{a}} x \\ \sqrt{\frac{b}{a}} x \\ \vdots \\ \sqrt{\frac{b}{a}} x\end{array}\right)$.

Therefore $\sqrt{a b}$ is an eigenvalue of $A(G)$. So $\sqrt{a b} \leq\left|\rho_{1}\right|$. By Lemma 2.3, we have

(2.14) $\sum_{j \in N_{i}^{+}} \rho_{1}\left(W_{i j}\right) \sum_{k \in N_{j}^{+}} \rho_{1}\left(W_{j k}\right)=\rho_{1}\left(W_{i}^{+}\right) \rho_{1}\left(W_{j}^{+}\right)=a b$ for $i \in V$.

By e15

$$
\left|\rho_{1}\right| \leq \max _{i \in V} \sqrt{\sum_{j \in N_{i}^{+}} \rho_{1}\left(W_{i j}\right) \sum_{k \in N_{j}^{+}} \rho_{1}\left(W_{j k}\right)}=\sqrt{a b} .
$$

Thus we obtain

$$
\left|\rho_{1}\right|=\sqrt{a b}=\max _{i \in V} \sqrt{\sum_{j \in N_{i}^{+}} \rho_{1}\left(W_{i j}\right) \sum_{k \in N_{j}^{+}} \rho_{1}\left(W_{j k}\right)} .
$$

Similarly one can easily see that $\left|\rho_{1}\right|=\rho_{1}\left(W_{i}^{+}\right)$for all $i$ for outweight regular digraph. Hence this completes the proof. 
Corollary 2.5. Let $G$ be a strongly connected weighted digraph. Then

$$
\left|\rho_{1}\right| \leq \max _{(i, j) \in E}\left\{\sqrt{\sum_{k \in N_{i}^{+}} \rho_{1}\left(W_{i k}\right) \sum_{k \in N_{j}^{+}} \rho_{1}\left(W_{j k}\right)}\right\}
$$

where $\left(W_{i j}\right)$ is the positive definite weight matrix of order $p$ of the arc $(i, j) \in E$. Moreover, the equality holds in e16 if and only if

(i) $G$ is an outweight regular digraph or $G$ is an outweight semi-regular digraph;

(ii) $\left\{W_{i j}\right\}_{i, j=1, \ldots, n}$ have a common eigenvector corresponding to the largest eigenvalue $\rho_{1}\left(W_{i j}\right)$ for all $i, j$.

Proof. For any vertex $i$ let $s$ be such that

$$
\sum_{k \in N_{s}^{+}} \rho_{1}\left(W_{s k}\right):=\max _{j \in N_{i}^{+}} \sum_{k \in N_{j}^{+}} \rho_{1}\left(W_{j k}\right) .
$$

Then we have

$$
\sum_{k \in N_{j}^{+}} \rho_{1}\left(W_{j k}\right) \leq \sum_{k \in N_{s}^{+}} \rho_{1}\left(W_{s k}\right)
$$

for any vertex $i, j \in N_{i}^{+}$. Thus, for any vertex $i$

$$
\begin{aligned}
\sum_{j \in N_{i}^{+}} \rho_{1}\left(W_{i j}\right) \sum_{k \in N_{j}^{+}} \rho_{1}\left(W_{j k}\right) & \leq \sum_{j \in N_{i}^{+}} \rho_{1}\left(W_{i j}\right) \sum_{k \in N_{s}^{+}} \rho_{1}\left(W_{s k}\right) \\
& \leq \max _{j \in N_{i}^{+}}\left\{\sum_{k \in N_{i}^{+}} \rho_{1}\left(W_{i k}\right) \sum_{k \in N_{j}^{+}} \rho_{1}\left(W_{j k}\right)\right\} .
\end{aligned}
$$

Therefore we get

$$
\left|\rho_{1}\right| \leq \max _{i \in V}\left\{\sqrt{\sum_{j \in N_{i}^{+}} \rho_{1}\left(W_{i j}\right) \sum_{k \in N_{j}^{+}} \rho_{1}\left(W_{j k}\right)}\right\}
$$




$$
\leq \max _{(i, j) \in E}\left\{\sqrt{\sum_{k \in N_{i}^{+}} \rho_{1}\left(W_{i k}\right) \sum_{k \in N_{j}^{+}} \rho_{1}\left(W_{j k}\right)}\right\} .
$$

From Theorem 2.4, the equality holds in e16 if and only if the conditions (i)-(ii) of the corollary hold for the digraph $G$.

Corollary 2.6. Let $G$ be a strongly connected weighted digraph where each arc weight $w_{i j}$ is a positive number. Then

$$
\rho_{1} \leq \max _{i \in V}\left\{\sqrt{w_{i}^{+} \bar{w}_{i}^{+}}\right\}
$$

where $\bar{w}_{i}^{+}=\sum_{j \in N_{i}^{+}} \frac{w_{i j} w j^{+}}{w_{i}^{+}}$. Moreover the equality holds in e17 if and only if $G$ is an outdegree regular digraph or $G$ is an outdegree semi-regular digraph.

Proof. For weighted digraph where the arc weight $w_{i j}$ is a positive number, we have $\rho_{1}\left(w_{i}^{+}\right)=w_{i}^{+}$and $\rho_{1}\left(w_{i j}\right)=w_{i j}$ for all $i, j$. Using Theorem 2.4, the result follows.

Corollary 2.7. [12]Let $G$ be a strongly connected unweighted digraph. Then

$$
\rho_{1} \leq \max _{i \in V}\left\{\sqrt{d_{i}^{+} m_{i}^{+}}\right\}
$$

where $d_{i}^{+}$and $m_{i}^{+}$is the outdegree and average 2-outdegree of the vertex $i$. Moreover the equality holds in e18 if and only if $G$ is an outdegree regular digraph or $G$ is an outdegree semi-regular digraph.

Proof. For unweighted digraph $w_{i j}=1$ and $w_{i}^{+}=d_{i}^{+}$. Using Corollary 2.6, we get the result.

From Corollary 2.5 we have the following corollary.

Corollary 2.8. Let $G$ be a strongly connected weighted digraph where each arc weight $w_{i j}$ is a positive number. Then

$$
\rho_{1} \leq \max _{(i, j) \in E}\left\{\sqrt{w_{i}^{+} w_{j}^{+}}\right\}
$$

where $w_{i}^{+}$is the sum of the weights of the arcs whose initial vertex is $i$. Moreover the equality holds in e19 if and only if $G$ is an outdegree regular digraph or $G$ is an outdegree semi-regular digraph. 
From Corollary 2.8 we have the following corollary.

Corollary 2.9. [12] Let $G$ be a strongly connected unweighted digraph. Then

$$
\rho_{1} \leq \max _{(i, j) \in E}\left\{\sqrt{d_{i}^{+} d_{j}^{+}}\right\}
$$

where $d_{i}^{+}$is the outdegree of the vertex $i$. Moreover the equality holds in e20 if and only if $G$ is an outdegree regular digraph or $G$ is an outdegree semi-regular digraph.

Remark 2.10. Obviously, for a weighted digraph $G$, we can define its inweight matrices, inweight regular digraph and inweight semi-regular digraph, etc. So we can easily obtain some similar results as in Theorem 2.4 and Corollary 2.5 for the spectral radius of $G$.

Acknowledgment. The authors thank Professor Charles R. Johnson for his helpful comments on Lemma 2.1. They are also thankful to TUBITAK and the Office of Selçuk University Scientific Research Project (BAP).

\section{References}

[1] D. M. Cvetković, M. Doob and H. Sachs, Spectra of Graphs, Academic Press, New York, 1980 (second revised ed., Barth, eidelberg, 1995).

[2] D. M. Cvetković, M. Doob , I. Gutman and A. Torgašev, Recent Results in the Theory of Graph Spectra, North-Holland, 1988.

[3] F. Buckley and F. Harary, Distance in Graphs, (Addison-Wesley, Redwood, 1990).

[4] G. H. Xu and C.Q. Xu, Sharp bounds for the spectral radius of digraphs. Linear Algebra Appl. 430, pp. 1607-1612, (2009).

[5] H. Minc, Nonnegative Matrices, Academic Press, New York, (1988)

[6] K. Ch. Das and R. . Bapat, A sharp upper bound on the spectral radius of weighted graphs. Discrete Math. 308, pp. 3180-3186, (2008). 
[7] K. Ch. Das, Extremal graph characterization from the bounds of the spectral radius of weighted graphs. Appl. Math. Comput. 217, pp. 7420-7426 (2011).

[8] K. Ch. Das and R.B. Bapat, A sharp upper bound on the largest Laplacian eigenvalue of weighted graphs. Linear Algebra Appl. 409, pp. 153-165 (2005).

[9] K. Ch. Das, Extremal graph characterization from the upper bound of the Laplacian spectral radius of weighted graphs, Linear Algebra Appl. 407, pp. 55-69 (2007).

[10] R. A. Horn and C.R. Johnson, Matrix Ananlysis, Cambridge University Press, New York, (1985).

[11] Ş.B. Bozkurt and A.D. Güngör, Improved bounds for the spectral radius of digraphs. Hacettepe J. Math. Stat. 39 (3), pp. 313-318, (2010).

[12] X. D. Zhang and J.S. Li, Spectral radius of nonnegative matrices and digraphs. Acta Math. Sin.18 (2), pp. 293-300, (2002).

\section{Ş. Burcu Bozkurt}

Department of Mathematics,

Science Faculty,

Selçuk University,

42075, Konya,

Turkey

e-mail : sbbozkurt@selcuk.edu.tr

and

\section{Durmuş Bozkurt}

Department of Mathematics,

Science Faculty,

Selçuk University,

42075, Konya,

Turkey

e-mail : dbozkurt@selcuk.edu.tr 